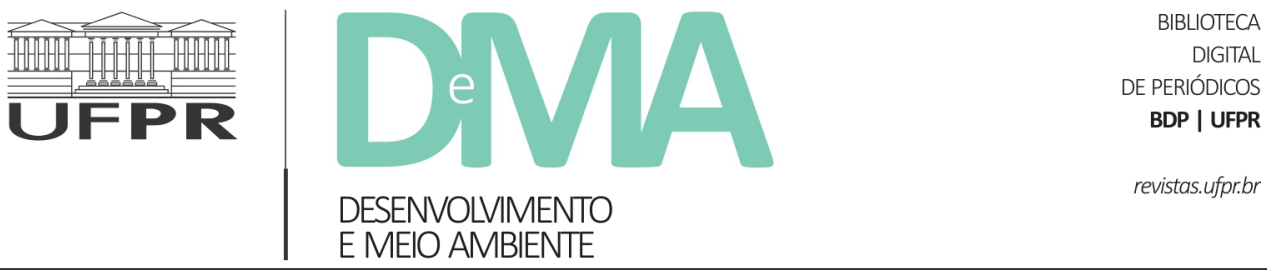

\title{
O Mecanismo Internacional de Varsóvia para Perdas e Danos: uma análise de seu primeiro ciclo
}

\section{Warsaw International Mechanism for Loss and Damage: a first cycle analysis}

\author{
Lívia Preti BOECHAT ${ }^{*}$, Wagner Costa RIBEIRO ${ }^{1}$ \\ ${ }^{1}$ Universidade de São Paulo (USP), São Paulo, SP, Brasil. \\ *E-mail de contato: li.pretiboechat@gmail.com
}

Artigo recebido em 1 de maio de 2020, versão final aceita em 11 de julho de 2021, publicado em 14 de dezembro de 2021.

RESUMO: $\quad$ Em um cenário marcado por modificações ambientais agudas, surge o Mecanismo Internacional de Varsóvia, o primeiro instrumento de cooperação internacional focado na minimização das perdas e danos inevitáveis associadas aos impactos das mudanças climáticas criado sob a égide da Convenção Quadro das Nações Unidas sobre Mudanças Climáticas (UNFCCC). Este artigo trata-se de uma avaliação de sua institucionalização e primeiro ciclo de funcionamento, entre 2013 e 2016, que, baseado em pesquisa documental, se inicia com uma discussão sobre o aumento do número e intensidade dos desastres relacionados ao clima, em seguida aborda as noções de perdas e danos por meio de revisão bibliográfica e, posteriormente, parte para a discussão do Mecanismo Internacional de Varsóvia, por meio do escrutínio de seu processo de criação, estruturação e implementação. Por fim, conclui-se que essa nova estrutura organizacional no âmbito da UNFCCC é mais uma alternativa complexa que se mostra de difícil implementação em um cenário muitas vezes adverso à cooperação internacional, inclusive para temas relevantes, como os que envolvem os impactos das mudanças climáticas.

Palavras-chave: mudanças climáticas; desastres; perdas e danos; mecanismo internacional de Varsóvia.

ABSTRACT: In a scenario marked by acute environmental changes, the Warsaw International Mechanism arises, the first international cooperation instrument focused on minimizing the inevitable losses and damages associated with the impacts of climate change created under the aegis of the United Nations Framework Convention on Climate Change (UNFCCC). This article is an evaluation of its institutionalization and first cycle of operation, between 2013 and 2016, which, based on documental research, it begins with a discussion about the increase in the number and intensity of weather-related disasters, then approaches the notions of loss and damage 
through a literature review, and, subsequently, goes on to the discuss the Warsaw International Mechanism, through the scrutiny of its creation, structuration and implementation processes. Lastly, the article concludes that this new organization structure within the United Nations Framework Convention on Climate Change (UNFCCC) is another complex alternative that is difficult to implement in a scenario that is often adverse to international cooperation, including for relevant issues, such as those involving the impacts of climate change.

Keywords: climate change; disasters; loss and damage; Warsaw international mechanism.

\section{Introdução}

No complexo cenário internacional, permeado por grandes mudanças ambientais de caráter transfronteiriço, a Convenção Quadro das Nações Unidas sobre Mudanças Climáticas (UNFCCC) se destaca por buscar combater, cooperativamente, as consequências negativas da interferência antrópica na dinâmica climática global. Porém, a velocidade com a qual as mudanças climáticas estão ocorrendo demonstrou a insuficiência não só das medidas de mitigação adotadas, mas também das ações adaptativas em impedir a concretização de seus impactos, cada vez mais severos e extensos. Segundo o IPCC (2019), verifica-se um aumento de desastres relacionados às mudanças climáticas nas últimas décadas, sendo que os principais envolvem tempestades e secas intensas, que afetam milhares de pessoas pelo mundo. O agravamento desse cenário leva não apenas a lamentáveis perdas de vidas humanas, mas também a prejuízos materiais, o que pode aumentar a demanda judicial por indenizações e lucros cessantes. Por isso é fundamental entender como a ordem ambiental internacional (Ribeiro, 2001) aborda a questão das perdas e danos residuais associadas aos impactos negativos da mudança do clima.
$\mathrm{O}$ artigo visa discutir o primeiro ciclo de vida do Mecanismo Internacional de Varsóvia para Perdas e Danos como último recurso criado pela UNFCCC para atenuar os impactos das mudanças do clima e suas consequências. Ele resulta de pesquisa documental que envolveu fontes oficiais eletrônicas e literatura científica. O resultado está expresso em cinco seções: o aumento dos desastres e suas consequências; discussão conceitual sobre perdas e danos; a institucionalização da demanda de perdas e danos na UNFCCC; a análise do Mecanismo Internacional de Varsóvia ao longo das COPs 19, 20, 21 e 22; e as considerações finais.

\section{O aumento dos desastres e suas consequências}

Conquanto não exista um consenso internacional sobre a definição de desastre, este artigo trabalhou, por sua vez, com o conjunto das definições da UNDRR (United Nations Office for Disaster Risk Reduction) e do banco de dados do CRED (Centre For Research on The Epidemiology of Disasters), o EM-DAT ${ }^{1}$ (The International Disaster Database). Embora se considere que há lacunas nas definições das instituições escolhidas, a UNDRR (2007) define desastre a partir da equação: (vulnerabilidade + ex-

\footnotetext{
${ }^{1}$ Existe uma ampla área de estudos dos desastres, com intensas discussões sobre seus elementos de definição pelos mais diversos vieses. Para maior aprofundamento no tema, consultar: Quarantelli (1985); Pelanda (1981); Dombrowsky (1981); Gilbert (1995); Valêncio et al. (2009); IPCC (2012).
} 
posição + hazard $) /$ capacidade $=$ desastre, enquanto o EM-DAT (2009) considera que um desastre deve preencher ao menos um destes critérios: dez ou mais pessoas mortas; cem ou mais pessoas afetadas (soma de pessoas que necessitam de assistência médica e abrigo); declaração de estado de emergência e/ou pedido de assistência internacional.

Baseado em tais definições, de acordo com o CRED (2015), a partir do final dos anos 1980, os desastres relacionados ao clima sofreram um aumento significativo e constante. Desde 2000, o EM-DAT, que é o maior banco de dados sobre desastres do mundo, registrou uma média de 341 desastres ao ano relacionados ao clima, representando um aumento de mais de 44\% em relação ao período de 1994 2000, e mais que dobrando o número de registros feitos entre 1980 - 1989 (CRED, 2015), destacando que, atualmente, os desastres relacionados ao clima correspondem a $90 \%$ de todos os desastres com origem em natural hazards (CRED \& UNDRR, 2016). O Programa das Nações Unidas para o Meio Ambiente (PNUMA, 2004), por sua vez, alerta que o número de desastres registrados não corresponde à realidade, pois muitos que incidem em escala local nos países em desenvolvimento não são reportados aos órgãos internacionais e, assim, não constam no banco de dados das maiores instituições que trabalham com a temática.

Por sua vez, a FAO (Organização das Nações Unidas para Agricultura e Alimentação) aponta que entre 2003 - 2013 as perdas econômicas causadas por desastres de deflagração por natural hazards somaram US\$550 bilhões e afetaram 2 bilhões de pessoas somente nos países em desenvolvimento. Entretanto, ressalta-se que o cálculo de perdas econômicas é subestimado, pois somente $35 \%$ dos desastres constantes no banco de dados do EM-DAT reportam esse tipo de informação (FAO, 2015; CRED \& UNDRR, 2016).

Embora parte do aumento registrado de desastres relacionados ao clima tenha sido ocasionada pelo aparecimento de centros de pesquisa, pelo desenvolvimento dos meios globais de comunicação, e pelo crescimento populacional, com concentração de capital e infraestrutura em áreas mais propensas à incidência de eventos extremos, houve um aumento real e significativo em sua frequência (CRED, 2015).

Duas causas principais são apontadas como responsáveis por esse aumento: a expansão das áreas de vulnerabilidade socioambiental, como resultado da combinação entre o crescimento urbano e a segregação socioespacial advinda do modelo hegemônico da economia (ISDR, 2008); e as mudanças climáticas.

Alega-se que as mudanças climáticas afetam o risco de desastres de duas formas principais: por meio do aumento do número e intensidade de eventos extremos relacionados ao clima ${ }^{2}$ e do desencadeamento e exacerbação de eventos de velocidade reduzida ${ }^{3}$ (PNUMA, 2004; Richardson et al., 2009; UNDRR, 2015). Nesse sentido, o quinto relatório

\footnotetext{
${ }^{2}$ Os eventos extremos são definidos como a ocorrência do valor de uma variável de tempo ou de clima acima ou abaixo de um limite, com um valor próximo às extremidades superiores ou inferiores do intervalo observado, podendo ser traduzidos na ocorrência de furacões, tempestades, secas, e ondas de calor, por exemplo (IPCC, 2012, p. 30).

${ }^{3}$ Os eventos de velocidade reduzida são progressivos e seus impactos aumentam com o passar do tempo, com projeções para afetar um número muito maior de pessoas. De acordo com a UNFCCC, os eventos de velocidade reduzida incluem: aumento do nível do mar; aumento das médias de temperatura; acidificação das águas oceânicas; retração dos glaciares; salinização; desertificação; degradação do solo e das florestas; e perda de biodiversidade (UNFCCC, 2011, p. 06).
} 
do Intergovernamental Panelon Climate Change (IPCC, 2014) alerta para o aumento já verificado, e desigualmente distribuído, de ondas de calor; secas mais longas e intensas; maior frequência e intensidade de precipitações; incremento do número de ciclones tropicais; aumento do nível médio do mar; retração de glaciares; e acidificação das águas oceânicas.

Segundo a FAO (2015), o acontecimento recorrente de desastres gera acúmulo de perdas e danos no setor agrícola, prejudicando o desenvolvimento nacional, causando impactos significativos na cadeia de alimentos, com consequências negativas nos fluxos de comércio de commodities agrícolas, e modificações do padrão de exportação e importação em curto e médio prazos. Consequentemente, a segurança alimentar também pode ser afetada devido à ocorrência de desastres, podendo levar à migração forçada de pessoas devido à falta de alimento. Ademais, a inviabilização ou destruição de recursos naturais e serviços ecossistêmicos, assim como as perdas de vida e de infraestrutura, também podem levar à migração forçada (GIZ, 2015), tanto interna quanto externamente, seja pela deterioração exacerbada dos recursos ambientais, seja pela pressão adicionada às tensões políticas e sociais. Neste cenário, o fluxo de migrantes transfronteiriços pode, ainda, causar o acirramento de tensões sociais ou mesmo adicionar mais pressão ao ambiente do território hospedeiro, levando à possível deflagração de conflitos e elevando a questão ao patamar da segurança nacional (Homer-Dixon, 1991).

Apesar das modificações ambientais terem funcionado até o momento mais como uma oportunidade e um estímulo à cooperação do que um desencadeador de conflitos no nível internacional, na esfera nacional ocorre a inversão dessa relação
(Dalby, 2016). Estudos envolvendo segurança ambiental estratégica apontam que as mudanças ambientais influenciam a deflagração de conflito por meio de seu impacto em quatro variáveis sociais: o declínio da economia, a diminuição da produção agrícola, a migração e o enfraquecimento das instituições autorizadas legítimas (Homer-Dixon, 1991; Nel \& Righarts, 2008; Atkins, 2016), sendo todas passíveis de serem influenciadas pela ocorrência de desastres de grandes proporções. Ademais, estudos recentes apontam que o estresse climático severo praticamente dobra o risco de deflagração de conflitos violentos (Levy, 2018). Torna-se pertinente não olvidar, contudo, que um desastre por si só não é capaz de desencadear um conflito violento, exigindo a existência prévia de vulnerabilidade estrutural e um vácuo de poder que seja incapaz de prover estabilidade (Atkins, 2016).

Nesse panorama, os pequenos Estados-Ilha, e os países costeiros em desenvolvimento, principalmente os localizados na África e na Ásia, mostram-se propensos a sofrerem maiores impactos. Tais países apresentam não só uma maior pré-disposição geográfica ao aumento do nível do mar e à ocorrência de desastres engatilhados por eventos extremos de clima, como também maior vulnerabilidade devido à falta de capital para investir em infraestrutura adequada e políticas de prevenção e alerta, à fraca governança de risco, e à dependência econômica muito grande da pesca e agricultura (UNDRR, 2015). Nesse panorama, a UNDRR (2015) alega que o risco de desastres já afeta a capacidade de alguns países em investir o capital necessário para se desenvolverem de forma sustentável, pois parte significativa do contingente que deveria ser destinado às políticas públicas está 
sendo direcionado para a recuperação e reconstrução das áreas atingidas.

Dessa forma, fica claro que a problemática do aumento dos desastres consiste em uma questão que envolve desde prejuízos materiais, ambientais e humanos, até possíveis ondas de desestabilização política e social, e, por isso, com o passar do tempo, a temática de perdas e danos conquistou espaço na agenda de negociações da maior convenção internacional sobre mudanças climáticas, a UNFCCC.

\section{Perdas e danos}

Mesmo que a questão de "perdas e danos" fosse constantemente suscitada desde o início dos anos 1990, por meio do AOSIS (Alliance of Small Island States), esse tema ganhou destaque somente na década de 2010, especialmente após a publicação do quinto relatório do IPCC (2014). Devido a sua aparição muito recente, ainda não há uma definição internacionalmente aceita a respeito do que são as perdas e danos, existindo diferentes perspectivas que digladiam para se tornarem legítimas.

Todavia, pode-se considerar que enquanto "dano" se refere aos impactos prejudiciais das mudanças climáticas que podem ser reparados ou reconstruídos, entende-se "perdas" como impactos prejudiciais que não são passíveis de reparo ou reconstrução (Schäfer \& Kreft, 2014; Thompson \& Otto, 2015). De acordo com McNamara (2014) e Dilley \& Grasso (2016), é imprescindível ressaltar que as perdas e danos relacionadas às mudanças do clima não se devem somente a estas, mas são determinadas por um conjunto de fatores no qual a vulnerabilidade desempenha um papel primordial, podendo ser traduzidas como o resultado do somatório: ocorrência de eventos extremos ou de velocidade reduzida + conjunto de elementos em risco (pessoas, estruturas, meios de subsistência) + características ambientais, econômicas, políticas e sociais que tornam determinado local/comunidade mais ou menos vulnerável. Ainda segundo Dilley \& Grasso (2016), enquanto os danos seriam os efeitos imediatos de um desastre, as perdas seriam seus efeitos em longo prazo.

A literatura sobre perdas e danos atribui, ainda, quatro características ao conceito: a inevitabilidade, a irreversibilidade (que pode ser física - como a perda de gelo permanente de glaciares - ou social - como a destruição de monumentos culturais), o fato de serem intoleráveis, e sua indução antrópica (Vulturius \& Davis, 2016). Quanto a esta última, enquanto há um amplo consenso em relação à atribuição dos eventos de velocidade reduzida às mudanças climáticas antrópicas, o mesmo não ocorre com os eventos extremos (Thompson \& Otto, 2015; Vulturius \& Davis, 2016), apesar de ser possível averiguar mudanças a respeito da probabilidade e da intensidade com que tais eventos têm ocorrido (Huggel et al., 2015).

Embora a UNFCCC não possua nenhum documento formal no qual conste a definição de perdas e danos, o SBI (Subsidiary Body for Implementation), um de seus principais órgãos subsidiários permanentes, a considera como "theactualand/ orpotentialmanifestationofimpactsassociatedwithclimatechange in developing countries that negatively affect humanand natural systems" (UNFCCC, 2012, p. 03). Nessa perspectiva, o termo se refere àqueles impactos que a mitigação não pode impedir e a adaptação não pode evitar (Harmeling et al., 2015; Mace \& Verheyen, 2016; Vulturius \& Davis, 2016), sendo essencial, destarte, diferenciar 
o escopo adaptativo e das perdas e danos, para que as medidas particulares a cada um possam ser adequadamente instituídas.

Nesse sentido, o PNUMA (GEAS \& UNEP, 2014) e o Instituto de Meio Ambiente e Segurança Humana da Universidade das Nações Unidas (UNU-EHS, 2016) consideram as perdas e danos como os efeitos negativos da variabilidade e das mudanças do clima com os quais as pessoas não foram capazes de lidar ou de se adaptar. Tais perdas e danos podem ocorrer tanto pela inevitabilidade de impactos devido à velocidade e intensidade com que as mudanças climáticas vêm ocorrendo, quanto pela ineficiência e inadequação de ações adaptativas causadas por falta de recursos, tecnologia, conhecimento, estabilidade e planejamento político (Harmeling et al., 2015; Mace \& Verheyen, 2016; Vulturius \& Davis, 2016).

Assim, embora ambas tenham como objetivo a diminuição dos impactos relacionados aos efeitos adversos das mudanças climáticas, a adaptação se desenvolve com base em uma perspectiva prospectiva, de prevenção aos impactos, enquanto a temática de perdas e danos aborda a questão por um viés retrospectivo, focado nas consequências dos impactos ou na sua inevitabilidade (Wallimann-Helmer, 2015; Bodansky, 2016). Dessa forma, suas esferas de atuação devem ser trabalhadas de forma integrada, porém diversa.

Apesar de grande parte das interpretações sobre o conceito de perdas e danos se focar somente na busca por compensação financeira imediata, ele carrega preocupações mais profundas e complexas, como perdas econômicas e não econômicas em médio e longo prazos. Tais perdas são causadas tanto por eventos extremos de clima como por aqueles de velocidade reduzida, envolvendo perdas nos serviços ecossistêmicos; perda de biodiversidade; perdas de meios de subsistência; perdas econômicas em longo prazo (como diminuição do turismo); perdas culturais (destruição de patrimônio cultural, templos, locais de culto, e sítios arqueológicos); perdas identitárias (impossibilidade de manutenção de determinado estilo de vida); perda de soberania, e problemas relacionados à migração devido à perda de território habitável (Hoffmaister et al., 2014; Harmeling et al., 2015; Thompson \& Otto, 2015).

Okereke (2006), Schäfer \& Kreft (2014) e Bodansky (2016) afirmam que as mudanças climáticas tornaram-se, portanto, uma questão de direitos humanos, por representarem uma ameaça à vida, à segurança física, à subsistência e à saúde, principalmente no que concerne às perdas e danos relacionadas a elas. Assim sendo, fica clara a importância que assume a criação e implementação de um mecanismo que seja capaz de tratar as perdas e danos associadas aos efeitos adversos das mudanças do clima nos países mais vulneráveis, notadamente nos insulares.

\section{A institucionalização da demanda de perdas e danos na UNFCCC}

Negociações envolvendo mudanças climáticas ocorrem desde a década de 1990. Apesar das controvérsias, surgiram instrumentos negociados que conformam uma estrutura organizacional que envolve muitos países com interesses nem sempre convergentes (Ribeiro, 2001; Ribeiro, 2010), sendo a Convenção Quadro das Nações Unidas sobre Mudanças Climáticas (UNFCCC), instituída em 1992 e comportando atualmente 197 países signatários, sua peça central. Desde as negociações sobre a 
criação da própria UNFCCC, o AOSIS já suscitava a necessidade da instituição de um mecanismo voltado para a abordagem de perdas e danos (Parker et al., 2015; Vulturius \& Davis, 2016), submetendo, em 1991, uma proposta reivindicando a criação de um fundo coletivo de seguro a ser incorporado na estrutura da Convenção, que fosse direcionado para a compensação financeira dos impactos causados pelo aumento do nível do mar devido às emissões de gases do efeito estufa (GEE) (Kugler \& Sariego 2016; Mace \& Verheyen, 2016).

A proposta não foi aceita e o tema só começou a ser considerado fora do grupo do AOSIS a partir da década de 2000, com a publicação do quarto relatório do IPCC, em 2007, que foi incisivo ao apontar que a mudança do clima era inequívoca e que muitos de seus impactos já seriam inevitáveis (Adaptation Committee, 2013). A partir desse documento, admitiu-se no âmbito da UNFCCC, pela primeira vez, a inevitabilidade da ocorrência de perdas e danos relacionadas aos efeitos adversos das mudanças climáticas, por meio da adoção do Plano de Ação de Bali como resultado da COP 13 (realizada naquele mesmo ano) (UNFCCC, 2008), que fazia parte de um conjunto de decisões tomadas com o intuito de representar todo o trabalho que deveria ser realizado pela UNFCCC e suas Partes, em diversas questões, na pavimentação de um caminho climaticamente seguro.

Na COP 14, o AOSIS submeteu nova proposta para o estabelecimento de um mecanismo voltado para o tratamento de perdas e danos nos SIDS (Small Island Developing States), nos LDCs
(Least Developed Countries), e em outros países em desenvolvimento particularmente vulneráveis, baseando a proposta em sete princípios do direito internacional do meio ambiente ${ }^{4}$ (AOSIS, 2008). O mecanismo teria como objetivo reduzir a vulnerabilidade e aumentar a capacidade adaptativa, sendo baseado em três componentes: seguro; reabilitação/compensação; e gerenciamento de risco (AOSIS, 2008). Além da proposta do AOSIS, uma série de documentos informais circularam durante as negociações, dentre os quais um artigo da WWF (World Wide Fund for Nature) intitulado Beyond Adaptation: The Legal Duty to Pay Compensation for Climate Change Damage (Vanhala \& Hestbaek, 2016), propondo ideias similares. Embora nenhuma delas tenha sido aceita, serviram para impulsionar a discussão, e o termo "compensação" começou a se disseminar em conversas informais nas negociações (Mace \& Verheyen, 2016).

Na COP 16, realizada em Cancun, em 2010, retomou-se o debate, que ficou marcado pela admissão oficial de que deveria ser atribuída a mesma importância às ações mitigativas e adaptativas, e se instituía o principal mecanismo voltado à adaptação submisso diretamente à Convenção, o Quadro de Adaptação de Cancun. O Quadro de Adaptação estabeleceu, sob seu escopo, o primeiro processo estrutural voltado à abordagem de perdas e danos: o Programa de Trabalho de Perdas e Danos (Adaptation Committee, 2013).

O Programa procurou abordar o enfrentamento de perdas e danos associadas aos impactos das mudanças climáticas nos países em desenvolvimento

\footnotetext{
${ }^{4}$ Os sete princípios citados na proposta: Responsabilidade de Não Causar Danos ao Meio Ambiente de Outros Estados ou Áreas Além de sua Jurisdição; $13^{\circ}$ princípio da Declaração do Rio; Poluidor-Pagador; Responsabilidades Comuns, Mas Diferenciadas; Precaução; Equidade; e Solidariedade Internacional (AOSIS, 2008, p. 2).
} 
mais vulneráveis aos seus efeitos adversos. Ele convidou as 197 Partes da Convenção (países-membro), e organizações relevantes, a submeterem visões e informações sobre os elementos que as mesmas acreditassem que deveriam constar no Programa de Trabalho (UNFCCC, 2011). Coube ao SBI, por sua vez, conduzir as atividades do Programa, bem como levar recomendações para discussão do tema na COP 18 (UNFCCC, 2016a). A COP 16 destacou-se, portanto, por ser a primeira vez em que a demanda para o enfrentamento de tal problema foi institucionalizada dentro da Convenção e entrava, obrigatoriamente, em sua agenda de trabalho.

A COP 18, realizada em Doha, capital do Qatar, em 2012, se tornou, então, um divisor de águas pela admissão inédita da Conferência das Partes de que a UNFCCC possui um papel fundamental no enfrentamento da problemática de perdas e danos (UNFCCC, 2013a). Essa vitória dos países em desenvolvimento e menos desenvolvidos mais vulneráveis às mudanças climáticas resultou de intensas negociações permeadas por discursos antagônicos em relação à polêmica temática da compensação. Naquele ano, a instituição de um mecanismo de compensação financeira por perdas e danos relacionadas aos efeitos adversos das mudanças do clima foi proposta pelo maior número de países até então, representados tanto por meio de frentes diversas (como o Grupo Africano, o AOSIS e o LDC), quanto de forma individual por várias delegações, como aquelas da Bolívia, Equador, Guatemala, China, El Salvador, Tailândia, Filipinas e Nicarágua (McNamara, 2014).

Em sua proposta, o AOSIS alegava que além das razões humanitárias que deveriam motivar a instituição de tal mecanismo compensatório, também era necessário levar em consideração a capacidade dos impactos climáticos desencadearem processos de decrescimento socioeconômico com o potencial de culminarem em situações de colapso social nos países mais vulneráveis, o que, devido à interdependência complexa que rege o sistema internacional, poderia se propagar para outras áreas por meio das densas conexões econômicas estabelecidas e dos deslocamentos humanos (AOSIS, 2012).

Enquanto, de um lado, os países em desenvolvimento mais vulneráveis reivindicavam a instituição de um mecanismo de compensação, de outro, os países desenvolvidos, liderados pelos EUA, se recusavam a atender tal demanda. Eles argumentavam que a implementação dessa proposta inibiria o planejamento e a execução de ações de adaptação, e que recursos teriam de ser desviados desse ramo de atuação para o segundo, além de colocarem a dificuldade, ou impossibilidade, de se valorar monetariamente as vidas, meios de subsistência e a renda de milhões de pessoas de diferentes países (Governo dos EUA, 2012 apud McNamara, 2014). Dessa forma, a compensação por perdas e danos se converteu em uma das questões mais controversas da COP 18.

De acordo com Huggel et al. (2015), um de seus defensores, a compensação é uma questão de direito, de política e, principalmente, de justiça climática, baseando-se, mesmo que sem menção direta, no discurso do conceito de dívida ecológica aplicada às questões climáticas. Assim sendo, a demanda pela criação de um mecanismo de compensação possui suas raízes originadas em, ao menos, três princípios do direito internacional: Responsabilidade de Não Causar Danos ao Meio Ambiente de outros Estados ou Áreas Além de sua Jurisdição; Poluidor-Pagador; e Responsabilidades Comuns, Mas Diferenciadas. Pode-se concluir, portanto, que 
a compensação por perdas e danos parte da premissa de que se as atividades de um país, ou de um grupo de países, causam danos a territórios além de sua jurisdição, gera-se a obrigação de efetuar as devidas ações de reparação, o que, no caso das mudanças climáticas, envolve a responsabilidade histórica desigual pelas emissões de GEE, e deveria incluir a compensação financeira, à luz da justiça climática.

Todavia, juridicamente qualquer ação de atribuição de responsabilidade procurando compensação deve demonstrar a causalidade entre o dano e a ação (ou omissão) que o causou (Thompson \& Otto, 2015; Vulturius \& Davis, 2016), sendo o ponto mais contencioso sobre o assunto. Dois obstáculos se destacam nessa tarefa:

(i) a impossibilidade de atribuição de perdas e danos exclusivamente às mudanças climáticas, pois os desastres responsáveis por causá-las ocorrem devido a um conjunto de fatores, incluindo elementos não-climáticos que podem elevar o nível de vulnerabilidade e exposição das sociedades (Wallimann-Helmer, 2015; Vulturius \& Davis, 2016), tais como o tipo de território ocupado, a constituição da infraestrutura física das construções locais, a ausência de medidas de prevenção ao risco de desastre e a falta de políticas públicas voltadas ao contingenciamento desse tipo de situação, o que é utilizado por países desenvolvidos para deslegitimar a demanda (McNamara, 2014), e

(ii) a impossibilidade de atribuir a ocorrência de eventos extremos individuais às mudanças do clima.

Ainda que seja impossível afirmar que determinado evento ocorreu devido às mudanças climáticas, a PEA (Probabilistic Event Attribution) já permite estabelecer a causalidade entre as mudanças climáticas e a mudança no fator de risco de ocorrência de eventos extremos (Parker et al., 2015; Thompson \& Otto, 2015). Ademais, ao considerar a Precaução como um dos princípios norteadores da UNFCCC, os países industrializados não deveriam utilizar a questão da atribuição como justificativa para se eximir de adotar a compensação financeira, pois as incertezas sobre determinado assunto não podem servir como justificativa para a omissão da ação por parte dos Estados. Entretanto, tal princípio foi convenientemente omitido das discussões sobre compensação.

Com imensa pressão dos países mais poderosos do sistema internacional, aqueles que haviam proposto o estabelecimento do mecanismo foram obrigados a recuar para que a temática continuasse avançando nas próximas conferências (Shamsuddoha \& Bijoy, 2013 apud McNamara, 2014). Com essa retração, os países desenvolvidos aceitaram estudar opções de arranjos institucionais para a criação de um mecanismo internacional de perdas e danos a ser estabelecido na COP 19 (UNFCCC, 2013a).

Apesar de insatisfatória, a COP 18 permitiu uma conquista inédita para o AOSIS e os LDCs, já que as Partes reforçaram a necessidade de fortalecer ações de cooperação internacional para entender e reduzir as perdas e danos, embora não tenha ficado claro como as ações seriam executadas. Ao final, as Partes também afirmaram que a falta de certeza científica não é justificativa para adiar a implementação de ações a esse respeito (UNFCCC, 2013a), consolidando a institucionalização da demanda. 


\section{O mecanismo internacional de Varsóvia para perdas e danos}

A seguir comentam-se as Conferências das Partes (COPs) da UNFCCC - órgão supremo executivo da Convenção que congrega todos os países que ratificaram ou aceitaram a Convenção (as Partes), podendo estar representados por delegações nacionais ou por organizações de integração econômica regional - que discutiram a criação do Mecanismo Internacional de Varsóvia para perdas e danos (WIM), o que permitirá conhecer suas atribuições.

\subsection{COP 19}

Na COP 19, realizada na Polônia, em 2013, os países em desenvolvimento mais vulneráveis aos efeitos adversos das mudanças do clima, em especial do grupo dos SIDS, AOSIS e do Grupo G77 + China $^{5}$, alegaram que a instituição de um mecanismo para tratar as perdas e danos era uma questão de direito e justiça, inclusive compensatória, pois não caberia a eles, contribuidores mínimos para o desencadeamento do problema, arcar com as consequências de uma crise climática com a qual não têm condições de lidar. Diferentemente dos outros anos, esse discurso ganhou forte impulso logo no primeiro dia de negociações devido ao emotivo depoimento do chefe da delegação Filipina, Naderev Saño, que expôs a situação calamitosa que se instaurou em seu país após a passagem do tufão Haiyan, apenas quatro dias antes do início da Conferência. Tal depoimento atraiu a atenção da mídia internacional para o tema durante as negociações, ajudando a pressionar os países desenvolvidos.
Aproveitando o momento favorável à discussão, o grupo do G-77 + China submeteu nova proposta para criar um mecanismo de perdas e danos, que as apresentava como um terceiro pilar da Convenção, ao lado da mitigação e adaptação. Ele deveria ser estabelecido de forma independente de outros mecanismos e submetido diretamente à Conferência das Partes (SBI, 2013). A proposta, apoiada pelo AOSIS, também demandava a criação de um novo fundo para financiar suas ações (SBI, 2013), e não foi bem recebida pelos países desenvolvidos (Thompson \& Otto, 2015).

A principal oposição à proposta concernia ao financiamento, pois os países desenvolvidos sabiam que a criação de um mecanismo independente exigiria também a adição de novos recursos para o estabelecimento e manutenção de suas atividades. Caso eles instituíssem o mecanismo de perdas e danos no âmbito do Quadro de Adaptação de Cancun, poderiam apenas remanejar os recursos existentes. Esse arranjo institucional submisso à adaptação é, todavia, passível de receber ao menos duas críticas: a instituição de medidas adequadas às perdas e danos não pode ocorrer em detrimento da adaptação, e seus escopos de atuação são distintos em sua essência.

Um relatório técnico do Secretariado da Convenção corroborou essa diferenciação de escopos de atuação ao pontuar que o custo total das mudanças do clima pode ser dividido em três:

(i) custos mitigativos;

(ii) custos adaptativos; e

(iii) custos de perdas e danos (UNFCCC, 2013b).

\footnotetext{
${ }^{5}$ Grupo fundado em 1964 que congrega atualmente 134 países em desenvolvimento (The Group of 77, 2021).
} 
Assim, a submissão ao Quadro de Adaptação não possuiria uma boa base argumentativa.

Os países desenvolvidos, todavia, não cederam às pressões. Apesar do texto de criação afirmar que "[...] loss and damage associated with the adverse effects of climat echange includes, and in some cases involves more than, that which can be reduced by adaptation" (UNFCCC, 2014a, p. 6), o Mecanismo Internacional de Varsóvia (WIM) para perdas e danos foi estabelecido sob o Quadro de Adaptação, o que indica que o mesmo foi instituído utilizando-se a técnica da ambiguidade construída, ou seja, ao mesmo tempo em que a Conferência das Partes reconhece que as perdas e danos vão além da adaptação, posicionam a existência do Mecanismo sob o Quadro de Adaptação. Essa manobra, aliada a uma definição vaga do objetivo geral do WIM, não permitiu especificar a constituição das perdas e danos, nem delimitar um escopo mínimo e/ou máximo pelo qual suas ações devem se pautar, contemplando a reivindicação dos países em desenvolvimento pela instituição de um mecanismo de perdas e danos e, concomitantemente, não comprometendo os países desenvolvidos com ações ou metas de financiamento.

Desse modo, o WIM foi criado com o objetivo de "[...] address loss and damage associated with the impacts of climate change, including extreme weather events and slow onset events, in developing countries that are particularly vulnerable to the adverse effects of climate change" (UNFCCC, 2014a, p. 06). Foi decidido que o Mecanismo deveria exercer três funções principais, que se complementam:

(i) melhorar o conhecimento e a compreensão das abordagens de gestão de risco para lidar com perdas e danos associadas aos efeitos adversos das mudanças climáticas;

(ii) fortalecer o diálogo, coordenação, coerência e sinergia entre os diversos stakeholders relevantes para a temática; e

(iii) empreender ações de suporte financeiro, tecnológico, e de capacitação para lidar com as perdas e danos advindos dos efeitos adversos das mudanças do clima (UNFCCC, 2014a).

Ademais, acordou-se que o WIM passaria por uma revisão na COP 22.

Embora tivesse sido solicitado aos países desenvolvidos o fornecimento de auxílio financeiro, tecnológico e de capacitação, não havia qualquer especificação sobre como tal auxílio seria fornecido ou a quantidade do mesmo (UNFCCC, 2014a). Como a resolução de criação deixou claro que todas as funções e atividades de implementação do Mecanismo estariam sujeitas à disponibilidade de recursos financeiros (UNFCCC, 2014a), ficou incerta sua capacidade de operacionalização.

A adoção do WIM dividiu opiniões quanto a sua real efetividade para enfrentar as perdas e danos. Para alguns, sua alocação sob o domínio da adaptação falhou ao não pontuar a questão de perdas e danos como o terceiro pilar da UNFCCC (McNamara, 2014); para outros, sua criação posicionou o tema como a terceira frente de ação do combate às mudanças do clima (Schäfer \& Kreft, 2014; Mece \& Verheyen, 2016). Em relação aos países em desenvolvimento, a maioria considerou insatisfatório o texto final de instituição do mecanismo devido à vaguidade de suas resoluções (ICTSD, 2013), pois o texto não definiu o que são perdas e danos, não as diferenciou da adaptação, não reconheceu a responsabilidade das economias industrializadas 
pelas consequências das mudanças climáticas, e não delineou nenhum plano nítido de ajuda (Calliari, 2014). Ademais, não foram instituídos fundos financeiros adicionais, nem foi definido como o Mecanismo seria financiado em longo prazo (Mace $\&$ Verheyen, 2016).

Apesar das críticas, sua existência legitimou a demanda sobre a necessidade de enfrentar as perdas e danos, e se comprometeu a fornecer e articular uma importante rede de conhecimento, compartilhamento de expertise, de coleta e disseminação de dados relacionados à abordagem de perdas e danos, além de melhorar o suporte técnico, o diálogo, e a cooperação entre os diferentes stakeholders dentro e fora da Convenção (Schäfer \& Kreft, 2014).

\subsection{COP 20}

Diferentemente do ano anterior, na COP 20 a questão de perdas e danos não recebeu muita atenção. Como resultados concretos, foram aprovados o plano de trabalho inicial de dois anos e a formação e atribuições de um Comitê Executivo permanente do WIM, estruturados durante o primeiro ano de sua criação.

Em relação ao Comitê, decidiu-se que ele seria composto por dez membros das Partes incluídas no então Anexo I e dez membros das Partes não incluídas no Anexo I, procurando uma representação equilibrada entre países desenvolvidos e em desenvolvimento. Já a elaboração do plano de trabalho contou com a participação ativa e relevante contribuição de inúmeros observadores de dentro e fora da Convenção, resultando em um plano inicial dividido em nove áreas de ação prioritária:
1 - análise de vulnerabilidade e de possíveis abordagens de perdas e danos;

2 - gerenciamento abrangente do risco;

3 - riscos e impactos de eventos de velocidade reduzida;

4 - abordagem das perdas não econômicas;

5 - análise da capacidade de preparo, resposta, construção de resiliência e recuperação;

6 - deslocamentos humanos associados aos impactos das mudanças do clima;

7- informação sobre instrumentos e ferramentas de financiamento do risco de perdas e danos;

8 - complementar e envolver organismos e grupos de expertise internos e externos à Convenção, nas atividades do Comitê Executivo;

9 - desenvolvimento de um plano de trabalho de cinco anos (UNFCCC, 2015).

Embora as críticas feitas à instituição do Mecanismo no ano anterior pudessem ser aplicadas também ao seu plano de trabalho inicial, sua adoção pode ser considerada um avanço. Um ponto positivo a ser destacado na estrutura do plano de trabalho se refere à ênfase dada aos processos de coleta de dados e aprofundamento do conhecimento sobre os vários aspectos que constituem as perdas e danos, especialmente considerando a limitada literatura existente sobre o tema. Além disso, a própria análise global de perdas e danos relacionadas aos efeitos adversos das mudanças climáticas pode funcionar como um instrumento de monitoração da eficiência de medidas de mitigação e de adaptação, servindo como fator de orientação de suas políticas.

Outro ponto relevante foi a abordagem, ainda que não da maneira desejada pelos países em desenvolvimento, da questão do financiamento. Apesar de não comportar qualquer tipo de medida 
de responsabilidade financeira baseada em justiça compensatória, a área de ação número 7 representou um modesto, mas relevante, avanço ao encorajar o gerenciamento compreensivo do risco por meio da difusão de informações relacionadas a instrumentos e ferramentas de financiamento do risco de perdas e danos, podendo incluir aspectos como: capacidade de gerenciamento compreensivo de risco baseado em compartilhamento e transferência; seguro de risco de catástrofe, financiamento de contingência, e financiamento de abordagens para desenvolvimento de resiliência climática (UNFCCC, 2014b). A sétima área compreendia, entre outras atividades, o encorajamento de instituições e fundos públicos bilaterais e multilaterais, e investidores privados, a incorporar o risco e a resiliência climática no desenvolvimento de projetos e nas decisões e critérios de investimento, além de se propor a encorajar, a promover e coordenar processos de pesquisa e desenvolvimento de instrumentos e ferramentas de financiamento que abordem os riscos de perdas e danos. Essa ação se mostra valiosa para os governos dos países em desenvolvimento que não sabem ao certo como esses mecanismos funcionam e podem ser utilizados, ou mesmo qual a extensão do sistema existente de seguro de risco de desastres relacionados ao clima, que corresponde, atualmente, a uma parcela significativa da economia global (McGeeet al., 2014). Nesse sentido, o plano de trabalho abre a possibilidade do estabelecimento de diálogo e coordenação com os seguros coletivos de base estatal que surgiram nos últimos anos, como o Caribbean Catastrophe Risk Insurance Facility (CCRIF), o Pacific Disaster Risk Financing and Insurance Program (PDRFIP), e o African Risk Capacity Insurance Company Limited (ARC).
Dessa forma, embora o plano de trabalho não tenha atendido todas as expectativas, ele conseguiu estabelecer as bases para a construção de uma resposta significativa da sociedade internacional à questão de perdas e danos relacionadas às mudanças do clima (Harmeling et al., 2015), com a maior preocupação imediata após a COP 20 consistindo na incorporação do tema no novo acordo a surgir em Paris.

\subsection{COP 21}

Na COP 21, em 2015, os países do grupo do AOSIS e do LDCs demandavam a inclusão de um organismo no novo acordo climático (a substituir o Protocolo de Kyoto) que prezasse pelo desenvolvimento de um sistema de tratamento mais pragmático da questão de perdas e danos, buscando o fortalecimento do WIM e a ampliação de seu mandato. Ademais, os países em desenvolvimento mais vulneráveis temiam que o WIM fosse esquecido ou descontinuado se a questão de perdas e danos não entrasse no novo Acordo (Mace \&Verheyen, 2016)

Os países desenvolvidos, por outro lado, tentaram evitar essa questão o quanto foi possível. A principal preocupação era de que a inserção de perdas e danos pudesse levar a demandas juridicamente respaldadas por compensação e reparação financeira, responsabilidade que os mesmos se recusavam a assumir, especialmente os EUA(Bodansky, 2016; Mace \& Verheyen, 2016). Para que estes se mostrassem mais abertos à inclusão da questão de perdas e danos no acordo, de modo separado da adaptação, os países que a reivindicavam chegaram a retroceder nos pontos mais contenciosos, a fim de garantir avanços posteriores que poderiam não 
ser obtidos caso o tema permanecesse excluído (Harmeling et al., 2015), repetindo o movimento de recuo que já havia sido executado na COP 18.

Após intensos debates, os países desenvolvidos cederam à pressão e concordaram em incluir o tema no Acordo de Paris, com a condição de que no documento final contendo as decisões da COP 21 fosse adicionado um parágrafo afirmando que a inclusão de um artigo referente a perdas e danos no Acordo não envolveria ou forneceria qualquer base para demandas de responsabilidade ou compensação (UNFCCC, 2016b). Mediante a adição dessa salvaguarda, foi dedicado um artigo inteiro às perdas e danos.

A sua inclusão, contudo, acabou ficando aquém daquilo que era esperado pelos países que a reivindicavam devido à falta de especificidade de suas ações. $\mathrm{O}$ artigo 8 , dedicado à questão, não estabeleceu ações mais pragmáticas para o enfrentamento do problema, e o parágrafo 51 da decisão que precedeu o Acordo descartou a possibilidade de qualquer tipo de auxílio financeiro baseado em responsabilidade e compensação, elementos que estão, de acordo com Hoad (2016), inextricavelmente ligados à luta por justiça. Todavia, essa não é uma decisão irreversível, pois nada impede que uma decisão posterior anule, altere ou detalhe a sua predecessora, se assim for decidido pelas Partes.

Apesar da composição do artigo 8 ter sido considerada como insatisfatória por aqueles países mais interessados no tema, a inclusão de perdas e danos e do WIM no Acordo de Paris é de grande importância por três motivos principais. O primeiro reside na admissão oficial de que adaptação e perdas e danos são temas distintos e, portanto, requerem tratamentos diferenciados (Bodansky, 2016). O segundo se refere ao entendimento e introdução de perdas e danos como constituindo o terceiro pilar de ação e cooperação climática da UNFCCC, embora ainda nem todos o considerem desta maneira (Vulturius \& Davis, 2016). E, por fim, sua inserção garante a busca por tratamentos adequados ao enfrentamento de perdas e danos e assegura a permanência do assunto na agenda de negociações (Harmeling et al., 2015).

Em relação ao plano de trabalho do WIM, o Comitê Executivo conseguiu apresentar avanços em seis áreas prioritárias (áreas 1, 3, 4, 5, 6 e 7), o que garantiu que a COP 21 decidisse oficialmente pela continuação do WIM além de seu período de revisão no ano seguinte. A COP de 2015 representou, portanto, um grande progresso no tratamento do tema de perdas e danos, na medida em que não só decidiu pela continuação do WIM, como incorporou o mesmo ao novo acordo climático negociado, o Acordo de Paris.

\subsection{COP 22}

A COP 22, por sua vez, organizada em 2016, ficou encarregada de fazer uma revisão do WIM, incluindo sua estrutura, mandato e eficácia, com base no trabalho empreendido até aquele momento pelo Comitê Executivo, que apresentou ao secretariado da $\mathrm{COP}$ o relatório anual de suas atividades.

Além do progresso obtido no plano inicial de trabalho de dois anos, aprovado na COP 20, apresentou-se um quadro indicativo de um plano de trabalho de cinco anos. O novo plano de trabalho foi estruturado em sete linhas gerais de ação estratégica:

(1) eventos de velocidade reduzida; 
(2) perdas não econômicas;

(3) abordagens de gerenciamento abrangente de risco para tratar e construir resiliência em longo prazo nos países, populações e comunidades mais vulneráveis às perdas e danos; e

(4) migração, deslocamento e mobilidade humana (UNFCCC, 2016c).

As outras três linhas estratégicas não foram definidas com precisão, porém já haviam sido reservadas para tratar sobre:

(5) financiamento;

(6) resultados adicionais do plano de trabalho de dois anos; e

(7) necessidades emergentes averiguadas (UNFCCC, 2016c).

A COP 22 representou um momento de transição para a questão de perdas e danos na medida em que, com a revisão feita, se encerrava o primeiro ciclo de vida do Mecanismo Internacional de Varsóvia e iniciava-se um novo, com a aprovação do quadro indicativo do plano de trabalho de cinco anos. Dessa forma, as Partes expressaram pública e oficialmente seu apreço pelo progresso feito na implementação de seu plano de trabalho inicial, enfatizando seu pioneirismo, estimulando a continuação de suas atividades e corroborando a importância do tema na agenda de negociações climáticas (UNFCCC, 2017). Ao mesmo tempo, determinou-se quais seriam os próximos passos a seguir, sinalizando que as áreas referentes às perdas não econômicas, aos eventos de velocidade reduzida, às abordagens de gerenciamento de risco, e às migrações e deslocamentos humanos seriam priorizadas.
Embora a priorização de tais áreas não fosse coincidente com os principais discursos que demandavam a instituição da abordagem de perdas e danos, que continuam se concentrando na necessidade de se estabelecer medidas de seguro e, principalmente, de compensação e reparação financeira aos países em desenvolvimento e menos desenvolvidos mais vulneráveis aos impactos dos efeitos adversos das mudanças climáticas, é inegável o avanço obtido na questão desde que surgiram as primeiras considerações oficiais a seu respeito, especialmente no que se refere à coleta e disseminação de informações, produção de conhecimento e compartilhamento de experiências. Ainda há um longo caminho a ser percorrido pelo WIM no tratamento de perdas e danos, mas que não será trilhado sozinho, pois uma de suas grandes qualidades consiste no caráter colaborativo de suas ações ao prezar pela participação dos mais diversos atores na formulação e implementação de suas metas e atividades, tanto no que concerne à participação das Partes, quanto das organizações relevantes para a temática de dentro e fora da Convenção, tais como o Comitê de Adaptação, o Comitê Executivo de Tecnologia, o Grupo de Especialistas dos Países Menos Desenvolvidos, o Fundo Especial de Mudanças Climáticas, e a Organização Internacional de Migração, dentre outros (UNFCCC, 2016c).

\section{Considerações finais}

A principal função do Mecanismo é, até o momento, organizar um repositório de informações a respeito das principais questões que emergem junto à concretização de perdas e danos associadas aos impactos das mudanças do clima. Isso se justifica 
em especial para avaliar temas complexos, como aqueles que concernem os eventos de velocidade reduzida e as perdas não econômicas, cujas fontes de informação são limitadas. O Mecanismo também é relevante por compartilhar boas práticas e lições aprendidas, como um instrumento que pode tanto evitar a proliferação de medidas ineficazes, quanto possibilitar a replicação de experiências bem-sucedidas.

Além disso, o Mecanismo passa a funcionar como uma plataforma de acesso e disseminação de informações sobre os instrumentos de seguro existentes no ramo dos riscos climáticos e como os mesmos podem ser utilizados para abordar a ocorrência de perdas e danos, função que se torna fundamental perante a atual multiplicação de seguros desse tipo. O mesmo também se propõe a estabelecer laços de cooperação e sinergia entre as organizações e agências dentro e fora da Convenção que trabalham com a temática, possibilitando a coordenação de atividades, o que permite identificar as principais lacunas no tratamento da questão e evitar a duplicidade de ações. Ademais, observa-se o direcionamento do foco atual para a problemática envolvendo os deslocamentos humanos associados aos impactos das mudanças climáticas, aspecto imprescindível na medida em que seu volume só tende a aumentar e não há proteção no direito internacional para as pessoas que o fazem, nem existe uma agência dentro do sistema da Organização das Nações Unidas especializada no tratamento da questão, o que torna o WIM um valioso instrumento no enfrentamento desse desafio.

Entretanto, ainda há muito a ser feito e diversas lacunas a serem preenchidas. Os seguros de risco climático existentes ainda são insuficientes para suprir a demanda dos países que deles necessitam e muitos países sequer têm condições de custear as coberturas oferecidas por tais seguros. Nesse sentido, o Mecanismo deveria criar um fundo de contingência financiado coletivamente, sem atribuição de responsabilidade por compensação que pudesse afastar os países industrializados, para atender as lacunas deixadas pelos instrumentos já existentes, que não só auxiliaria diretamente os países mais vulneráveis em sua recuperação interna, mas também contribuiria para evitar a conformação de situações que podem vir a se tornar fatores de desestabilização, como o acirramento de tensões e conflitos e as migrações de caráter climático. Além disso, o custeio da manutenção do próprio WIM e de suas respectivas atividades carece de melhor definição e a alocação do Mecanismo sob o Quadro de Adaptação de Cancun deveria ser revista.

Apesar de ainda ter um longo caminho pela frente, sua instituição representa a resposta da comunidade internacional ao inegável aumento do número e intensidade dos desastres de origem climática, consistindo na mais recente tentativa de atenuação dos impactos inevitáveis das mudanças do clima.

\section{Agradecimentos}

Este artigo só pôde ser produzido graças ao apoio da Coordenação de Aperfeiçoamento de Pessoal de Nível Superior (CAPES) e do Instituto de Energia e Ambiente da Universidade de São Paulo (IEE - USP). 


\section{Referências}

Adaptation Commitee of United Nations Framework Convention on Climate Change. The State of Adaptation under the United Nations Framework Convention - 2013 thematic report, 2013. Disponível em: < https://unfccc.int/ files/adaptation/cancun_adaptation_framework/adaptation_committee/application/pdf/ac_2013_report_low_res. pdf $>$. Acesso em: jul. 2017.

AOSIS - Alliance of Small Island States. Proposal to the AWG-LCA Multi-Window Mechanism to Address Loss and Damage from Climate Change Impacts, 2008. Disponível em: $<$ http://unfccc.int/files/kyoto_protocol/application/pdf/ aosisinsurance061208.pdf>. Acesso em: jun. 2017.

AOSIS - Alliance of Small Island States. Views and information from Parties and relevant organizations on the possible elements to be included in the recommendations on loss and damage in accordance with decision 1/CP.16, 2012. Disponível em: $<$ https://unfccc.int/sites/default/files/ resource/docs/2012/sbi/eng/misc14a02.pdf > . Acesso em: jul. 2017.

Atkins, E. Environmental Conflict: a misnomer? In: Sosa-Nunez, G.; Atkins, E. (Eds). Environment, Climate Change and International Relations. Bristol: E-International Relations Publishing, 99-114, 2016.

Bodansky, D. The Paris Climate Change Agreement: a new hope? American Journal of International Law, 110 (2), 288319, 2016. doi: 10.5305/amerjintelaw.110.2.0288

Calliari, E. Loss and Damage: a critical discourse analysis. Fondazione Eni Enrico Mattei; Euro-Mediterranean Center on Climate Change, 2014. Disponível em: https://papers. ssrn.com/sol3/papers.cfm?abstract_id=2510785

CRED - Centre for Research on the Epidemiology of Disasters. Human Cost of Natural Disasters: a global perspective, 2015. Disponível em: <https://www.emdat.be/ human_cost_natdis $>$. Acesso em: fev. 2017.

CRED - Centre for Research on the Epidemiology of Disasters; UNDRR - United Nations Office for Disaster Risk Reduction. The Human Cost of Weather Related Disasters 1995-2015, 2016. Disponível em: <https://www.UNDRR.
org/2015/docs/climatechange/COP21_WeatherDisastersReport_2015_FINAL.pdf>. Acesso em: ago. 2017.

Dalby, S. Environment and International Politics: linking humanity and nature. In: Sosa-Nunez, G.; Atkins, E. (Eds.). Environment, Climate Change and International Relations. Bristol: E-International Relations Publishing, p. 42-59, 2016.

Dilley, M.; Grasso, V. F. Disaster reduction, loss and damage data, and the post-2015 international policy agenda. Environmental Science \& Policy, 61, 74-76, 2016. doi: 10.1016/j.envsci.2016.04.002

Dombrowsky, W. R. Another Step Toward a Social Theory of Disaster. Disaster Research Center, University of Delaware, 1981. Disponível em: < https://udspace.udel.edu/bitstream/ handle/19716/442/PP70.pdf? sequence=3\&isAllowed $=\mathrm{y}>$. Acesso em: mai. 2017.

EM-DAT - The International Disaster Database. Explanatory Notes, 2009. Disponível em: <https://www.emdat.be/ explanatory-notes $>$. Acesso em: jul. 2018.

FAO - Food and Agriculture Organization of the United Nations. The Impact of Natural Hazards and Disasters on Agriculture, Food Security and Nutrition, 2015. Disponível em: <http://www.fao.org/3/i5128e/i5128e.pdf>. Acesso em: mai. 2017.

GEAS - Global Environmental Alert Services; UNEP United Nations Environment Programme. Loss and damage: when adaptation is not enough, 2014. Disponível em: $<$ https://na.unep.net/geas/archive/pdfs/geas_apr2014_climate_change.pdf $>$. Acesso em: fev. 2017.

Gilbert, C. Studying Disaster: a review of the main conceptual tools. International Journal of Mass Emergencies and Disasters, 13 (3), 231-240, 1995.

GIZ - Deutsche Gesellschaft Fur Internationale Zusammenarbeit. Climate Risk Insurance for strengthening climate resilience of poor people in vulnerable countries: a background paper on challenges, ambitions and perspectives, 2015. Disponível em: <https://www.giz.de/de/downloads/ giz-2016-en-climate_risk.pdf>. Acesso em: mai. 2017.

Harmeling, S.; Chamling Rai, S.; Singh, H.; Anderson, T. Loss and Damage: climate reality in the 21 st century, 2015. 
Disponível em: $<$ https://actionaid.org/sites/default/files/ loss_and_damage_-_climate_reality_in_the_21st_century. pdf>. Acesso em: abr. 2017.

Hoad, D. The 2015 Paris Climate Agreement: outcomes and their impacts on small island states. Island Studies Journal, 11 (1), 315-320, 2016.

Hoffmaister, J. P.; Talakai, M; Damptey, P; Barbosa, A. S. Warsaw International Mechanism for loss and damage: Moving from polarizing discussions towards addressing the emerging challenges faced by developing countries, 2014. Disponível em: < https://www.twn.my/title2/climate/ info.service/2014/cc140101/Opinion\%20LD-060114.pdf>. Acesso em: jan. 2016.

Homer-Dixon, T. On the Threshold: environmental changes as causes of acute conflicts. International Security, 16 (2), 76-116, 1991. Disponível em: https://homerdixon.com/ on-the-threshold-full-article/

Huggel, C; Stone, D; Eicken, H; Hansen, G. Potential and limitations of the attribution of climate change impacts for informing loss and damage discussions and policies. Climate Change, 133, 453-467, 2015. doi: 10.1007/s10584015-1441-z

ICTSD - International Centre for Trade Sustainable Development. Controle de Perdas e Danos em Varsóvia, 2013. Disponível em: <https://ictsd.iisd.org/sites/default/files/ news/2013/11/boletim-pontes-varsovia-2.pdf $>$. Acesso em: mai. 2017.

IPCC - Intergovernamental Panel on Climate Change. Managing the Risks of Extreme Events and Disasters to Advance Climate Change Adaptation, 2012. Disponível em: $<$ https:// www.ipcc.ch/report/managing-the-risks-of-extreme-events-and-disasters-to-advance-climate-change-adaptation/>. Acesso em: jul. 2017.

IPCC - Intergovernamental Panel on Climate Change. Climate Change 2014: Synthesis Report - Contribution of Working Groups I, II and III to the Fifth Assesment Report of the Intergovernamental Panel on Climate Change, 2014. Disponível em: <https://www.ipcc.ch/report/ar5/syr/>. Acesso em: mai. 2017.

IPCC - Intergovernamental Panel on Climate Change. Climate Change and Land: an IPCC special report on climate change, desertification, land degradation, sustainable land management, food security, and greenhouse gas fluxes in terrestrial ecosystems, 2019. Disponível em: $<$ https://www. ipcc.ch/srccl/>. Acesso em: abr. 2021.

ISDR - International Strategy for Disaster Reduction. Climate Change and Disaster Risk Reduction, 2008. Disponível em: $<$ https://eird.org/publicaciones/Climate-Change-DRR. pdf $>$. Acesso em: mai. 2017.

Kugler, N. R.; Sariego, P. M. "Climate change damages", conceptualization of a legal notion with regard to reparation under international law. Climate Risk Management, 13, 103-111, 2016. doi: 10.1016/j.crm.2016.06.004

Levy, M. Natural Resources and Conflict Causes - Drivers of Violent Conflict Emergency. Environmental Security and Sustaining Peace Course, SDG Academy, 2018. Disponível em: $<$ https://sdgacademy.org/course/environmental-security-and-sustaining-peace/>. Acesso em: mai. 2018.

Mace, M. J.; Verheyen, R. Loss, Damage and Responsibility after COP21: All Options Open for the Paris Agreement. Review of European Community \& International Environmental Law (Reciel), 25 (2), 197-214, 2016. doi: 10.1111/ reel.12172

McGee, J.; Phelan, L.; Wenta, J. Writing the Fine Print: Developing Regional Insurance for Climate Change Adaptation in the Pacific. Melbourne Journal of International Law, 15, 444-472, 2014.

McNamara, K. E. Exploring Loss and Damage at the International Climate Change Talks. International Journal of Disaster Risk Science, 5, 242-246, 2014.

Nel, P.; Righarts, M. Natural Disasters and the Risk of Violent Civil Conflict. International Studies Quartely, 52 (1), 159-185, 2008. doi: 10.1111/j.1468-2478.2007.00495.x

Okereke, C. Global environmental sustainability: Intragenerational equity and conceptions of justice in multilateral environmental regimes. Geoforum, 37, 725-738, 2006. doi: 10.1016/j.geoforum.2005.10.005.

Parker, H. R; Cornforth, R; Boyd, E; James, R; Otto, F; Allen, M. Implications of Event Attribution for Loss and Damage Policy. Weather, 70 (9), 268-273, 2015. doi: 10.1002/wea.2542 
Pelanda, C. Disaster and Socio systemic Vulnerability. Disaster Research Center, University of Delaware, 1981. Disponível em: <https://udspace.udel.edu/bitstream/ handle/19716/440/PP68.pdf? sequence=3\&isAllowed=y $>$. Acesso em: mar. 2017.

PNUMA - Programa das Nações Unidas para o Meio Ambiente. Estado do Meio Ambiente e Retrospectivas Políticas: 1972-2002, 2004. Disponível em: <http://vampira.ourinhos. unesp.br:8080/cediap/material/o_estado_do_meio_ambiente_e_retrospectivas_politicas_-_artigo_do_site_ibama_capitulo_2.pdf $>$. Acesso em: fev. 2017.

Quarantelli, L. Whatis a disaster? The need for clarification in Definition and Conceptualization in Research. Disaster Research Center, 1985. Disponível em: <https://udspace. udel.edu/bitstream/handle/19716/1119/ART177.pdf?sequence $=6 \&$ isAllowed $=\mathrm{y}>$. Acesso em: mar. 2017 .

Ribeiro. W. C. A Ordem Ambiental Internacional. São Paulo: Contexto, 2001.

Ribeiro, W. C. Geografia política e gestão internacional dos recursos naturais. Estudos Avançados, 24 (68), 69-80, 2010.

Richardson, K.; Steffen, W.; Schellnhuber, H.; Alcamo, J.; Barker, T.; Kammen, D.; Leemans, R.; Liverman, D.; Munasinghe, M.; Osman-Elasha, B.; Stern, N.; Waever, O. Climate Change: global risks, challenges and decisions, 2009. Disponível em: $<$ https://globe.ku.dk/staff-list/?pure=en\%2Fpublications\%2Fclimate-change--global-risks-challenges--decisions(378cecf0-805d-11de-8bc9-000ea68e967b). html>. Acesso em: mai. 2017.

SBI - Subsidiary Body for Implementation. Submission from the Group of 77 and China, 2013. Disponível em: $<$ http://unfecc.int/resource/docs/2013/sbi/eng/crp01.pdf $>$. Acesso em: jun. 2017.

Schäfer, L.; Kreft, S. Loss and Damage: Roadmap to Relevance for the Warsaw International Mechanism, 2014. Disponível em: $<$ https://germanwatch.org/en/8366>. Acesso em: abr. 2017.

Thompson, A.; Otto, F. E. L. Ethical and Normative Implications of Weather Event Attribution for Policy Discussions Concerning Loss and Damage. Climatic Change, 133, 439451, 2015. doi: 10.1007/s10584-015-1433-z
The Group of 77. About the Group of 77, 2021. Disponível em: <https://www.g77.org/doc/>. Acesso em: jun. 2021.

UNDRR - United Nations Office for Disaster Risk Reduction. Terminology, 2007. Disponível em: <https://www. undrr.org/terminology/disaster-risk>. Acesso em: jan. 2016.

UNDRR - United Nations Office for Disaster Risk Reduction. Making Development Sustainable: The Future of Disaster Risk Management, 2015. doi: 9789211320428

UNFCCC - United Nations Framework Convention on Climate Change. Report of the Conference of the Parties on its thirteen session, held in Bali from 3 to 15 December 2007, 2008. Disponível em: <http://unfccc.int/resource/ docs/2007/cop13/eng/06a01.pdf>. Acesso em: jan. 2016.

UNFCCC - United Nations Framework Convention on Climate Change. Report of the Conference of the Parties on its Sixteen Session, Held in Cancun from 29 November to 10 December 2010, 2011. Disponível em: <http://unfccc. int/resource/docs/2010/cop16/eng/07a01.pdf > . Acesso em: jan. 2016.

UNFCCC - United Nations Framework Convention on Climate Change. A literature review on the topics in the context of thematic area 2 of the work programme on loss and damage: a range of approaches to address loss and damage associated with the adverse effects of climate change, 2012. Disponível em: <http://unfccc.int/resource/docs/2012/sbi/ eng/inf14.pdf>. Acesso em: jun. 2017.

UNFCCC - United Nations Framework Convention on Climate Change. Report of the Conference of the Parties on its eighteenth session, held in Doha from 26 November to 8 December 2012, 2013a. Disponível em: $<$ http://unfccc. int/resource/docs/2012/cop18/eng/08a01.pdf>. Acesso em: jan. 2016.

UNFCCC - United Nations Framework Convention on Climate Change. Non-economic losses in the context of the work programme on loss and damage, 2013b. Disponível em: <http://unfccc.int/resource/docs/2013/tp/02.pdf>. Acesso em: jun. 2017.

UNFCCC - United Nations Framework Convention on Climate Change. Report of the Conference of the Parties on its nineteenth session, held in Warsaw from 11 to 23 November 2013, 2014a. Disponível em: <http://unfccc.int/resource/ 
docs/2013/cop19/eng/10a01.pdf>. Acesso em: jan. 2016.

UNFCCC - United Nations Framework Convention on Climate Change. Report of the Executive Committee of the Warsaw International Mechanism for Loss and Damage associated with Climate Change Impacts, 2014b. Disponível em: <http://unfccc.int/resource/docs/2014/sb/eng/04.pdf> Acesso em: jun. 2017.

UNFCCC - United Nations Framework Convention on Climate Change. Report of the Conference of the Parties on its twentieth session, held in Lima from 1 to 14 December 2014, 2015. Disponível em: <http://unfccc.int/resource/ docs/2014/cop20/eng/10a02.pdf>. Acesso em: jan. 2016.

UNFCCC - United Nations Framework Convention on Climate Change. Chronology $-L \& D$ workstream, 2016 a. Disponível em: $<$ https://unfecc.int/topics/adaptation-and-resilience/workstreams/loss-and-damage-ld/chronology-ld-workstream\#eq-8>. Acesso em: jan. 2016.

UNFCCC - United Nations Framework Convention on Climate Change. Report of the Conference of the Parties on its twenty-first Session, held in Paris from 30 November to 13 December 2015, 2016b. Disponível em: <http://unfccc. int/resource/docs/2015/cop21/eng/10a01.pdf > . Acesso em: jan. 2016.

UNFCCC - United Nations Framework Convention on Climate Change. Report of the Executive Committee of the Warsaw International Mechanism for Loss and Damage associated with Climate Change Impacts, 2016c. Disponível em: $<$ http://unfccc.int/resource/docs/2016/sb/eng/03.pdf $>$. Acesso em: jul. 2017.
UNFCCC - United Nations Framework Convention on Climate Change. Report of the Conference of the Parties on its twenty-second session, held in Marrakech from 7 to 18 November 2016, 2017. Disponível em: <http://unfccc. int/resource/docs/2016/cop22/eng/10a01.pdf > . Acesso em: jul. 2017.

UNU-EHS - Institute for Environment and Human Security. Loss and damage livelihood resilience, 2016. Disponível em: <http://icccad.net/wp-content/uploads/2016/11/Loss and_damage_Policy_Brief_10.pd $>$. Acesso em: mai. 2018.

Valêncio, N.; Siena, M.; Marchezini, V.; Gonçalves, J. Sociologia dos Desastres: construção, interfaces e perspectivas no Brasil. São Carlos: RiMa Editora, 2009.

Vanhala, L.; Hestbaek, C. Framing Climate Change Loss and Damage in UNFCCC Negotiations. Global Environmental Politics, 16 (4), 111-129, 2016. doi: 10.1162/ GLEP_a_00379

Vulturius, G.; Davis, M. Defining loss and damage: the science and politics around one of the most contested issues within the UNFCCC, 2016. Disponível em: <https:// mediamanager.sei.org/documents/Publications/Climate/ SEI-DB-2016-Loss-and-damage-4-traits.pdf $>$. Acesso em: mai. 2017.

Wallimann-Helmer, I. Justice for Climate Loss and Damage. Climatic Change, 133, 469-480, 2015. doi: 10.1007/ s10584-015-1483-2 